\title{
Dynamics of Amphiphilic Surfactants Confined in Montmorillonite Slits with Different Cation Exchange Capacities
}

\author{
Georgios Polizos, ${ }^{*},{ }^{\dagger}$ Richard A. Vaia, ${ }^{\ddagger}$ Hilmar Koerner, ${ }^{\ddagger}$ and Evangelos Manias ${ }^{*}, \ddagger$ \\ $\dagger$ Oak Ridge National Laboratory, Oak Ridge, Tennessee 37831, United States \\ ${ }^{\ddagger}$ Air Force Research Laboratory, Materials and Manufacturing Directorate, Wright-Patterson Air Force Base, Ohio 45433, United \\ States \\ ${ }^{\S}$ Department of Materials Science and Engineering, Pennsylvania State University, University Park, Pennsylvania 16802, United States
}

ABSTRACT: Alkylammonium surfactants were nanoscopically confined between montmorillonite layers of varied negative surface charge, i.e., of varied cation exchange capacities. Dielectric relaxation spectroscopy was employed to probe the interfacial dynamics, arising from the mobility of the cations on the silicate surfaces, as a function of the confining walls' surface charge. Standard methods to dry the organo-silicates were employed; however, water was still detected physisorbed in the galleries; the dynamics of these water molecules were also detected and were found to be coupled with the dynamics of the ammoniums at high temperatures. A transition in the mobility of the cations, approximately at $75{ }^{\circ} \mathrm{C}$ (which is in good agreement with the conformational changes of the alkyl groups, reported in the literature), is observed, and a model is proposed on the basis of the dynamics of the confined water. Entropic contributions arising from variation in the surface charge density are also discussed in detail. The existence of water in the interlayer-in spite of drying the nanofillers in accordance to industrial practices-and the interrelated surfactant/ water dynamics bring forward important implications for the design and processing of polymer-based nanocomposites based on these fillers.

\section{INTRODUCTION}

In the last two decades, organically modified layered silicates (mica-type, i.e., 2:1 alumino-phyllosilicates) dispersed in polymers, termed as nanocomposites, have attracted considerable renewed scientific attention, because of their remarkable enhancements in mechanical, barrier, and flame retardancy properties. ${ }^{1}$ Frequently, alkylammonium surfactants are used for surface modification of such nanoparticles, where dispersion in an organic matrix is required: Specifically, pristine silicates are modified (ionically exchanged by alkylammoniums) prior to mixing with a polymer matrix, since appropriate selection of the cationic surfactant lowers the attractive interaction energy between the inorganic layers, and leads to a thermodynamically favorable dispersion in polymers. ${ }^{2,3}$ Several investigations have been carried out on the intercalated surfactants in an effort to improve the polymer/inorganic compatibility. Fundamental studies have also probed the unique spatial configuration of the amphiphile cationic alkanes when nanoscopically confined $(\sim 1$ $\mathrm{nm}$ ) and physisorbed on silicate surfaces (this sorption is mediated by Coulombic attraction between the organic surfactant cations and the negatively charged silicates).

The dynamics of nanoconfined surfactant tails, mostly paraffins, are crucial for the kinetics of nanocomposite formation $^{4,5}$ and, as such, have been studied in detail by both molecular dynamics computer simulations ${ }^{6-11}$ and experimental techniques. ${ }^{12-16}$ At elevated temperatures, depending on the alkyl length and surfactant packing density, kinetic first order phase transitions were detected for these nanoscopically confined surfactants. These transitions are a result of the alkyl chain conformational changes. Such conformational changes manifest in two kinds of structural rearrangements for the nanoconfined surfactant layer: (a) For high grafting densities (high CEC values, typical of micas and high-CEC vermiculites), there is a continuous increase in the average tilt angle of the paraffinic-type surfactant arrangement within the silicate intergallery. ${ }^{17}$ (b) For lower grafting densities (midrange CEC values, as the ones studied here, typical of montmorillonites and hectorites), there is a stepwise change from a monolayer to a bilayer with increasing surfactant molecular volume ${ }^{6-11,17}$ (e.g., with increasing alkyl chain length for alkyl-ammoniums or changes from primary alkyl-amines to quaternary multialkyl amines), followed by a "multilayer" formation for even larger surfactants. ${ }^{17}$ These structural rearrangements of the surfactant layer are associated with configurational changes of the alkyl chains, typically trans/ gauche ratio changes, due to steric (molecular packing) effects as the grafting density (viz., CEC) is altered. Also, from a dynamics viewpoint, similar trans/gauche changes occur during the dynamic transition, toward more disordered or "liquid" states, at higher temperatures. A second, albeit less substantial,

Received: July 9, 2013

Revised: September 30, 2013

Published: October 1, 2013 
mechanism relates to the ammonium cation (headgroup) dynamics. Similar to the conductivity mechanism of alkali metal in glassy oxides, ${ }^{18,19}$ when the ammonium cations have sufficient thermal energy, it is possible to be displaced from their initial potential well minima to neighboring ones. These minima are defined by the electrostatic interactions with the negatively charged crystal surface and typically give rise to a hopping diffusion on the silicate surface. ${ }^{20}$ In addition, this translational surfactant motion is usually assisted by surface adsorbed water, which can be removed only at very high temperatures $^{21}$ (much higher than the boiling point of water and higher than the degradation temperature of most organic moieties), and thus, the existence and dynamics of water should also be considered in this context.

Molecular dynamics simulations, performed in the absence of water, revealed the ability of octadecyl-trimethyl-ammonium ions to rearrange on the silicate surfaces at high temperatures. ${ }^{6}$ Experimentally, Jacobs et al. ${ }^{22}$ studied the same dynamics in alkyl quaternary ammonium exchanged montmorillonite (MMT) by employing impedance spectroscopy. The authors suggested a correlation between the Vogel-Tammann-Fulcher (VTF) type dynamics of the surfactant cationic groups and the transitions of the alkyl tails, in good agreement with differential scanning calorimetry (DSC) results. In related investigations, the headgroup and tail dynamics were identified by applying electron paramagnetic spectroscopy (EPR) along with solidstate NMR spectroscopy. ${ }^{23-25}$

In this paper, we present a comprehensive approach for the mobility of the intercalated cationic surfactants. Broadband dielectric relaxation spectroscopy (DRS) was employed to investigate the mobility of alkylammonium cations with an alkyl tail of 18 carbon atoms (octadecylammonium) that are intercalated between montmorillonite (MMT) platelets. We consider tangential cationic diffusion at a double layer (silicate/ water) interface. This diffusion is restricted by the silicate geometry and results in a surface polarization. The mobility of the ammonium cations was found to depend on the silicate surface charge density (typically quantified as the silicate's cation exchange capacity, CEC) and on the dynamics of the confined water. The effects of these two contributions are discrete, and are discussed here in detail.

\section{EXPERIMENTAL SECTION}

Materials. Two different cation exchange capacity (CEC) montmorillonites were prepared by annealing the same $\mathrm{Li}^{+}$ exchanged montmorillonite (purified PGV, Nanocor Inc.) at 120 and $130{ }^{\circ} \mathrm{C}$ under a vacuum for $24 \mathrm{~h}$, following typical reduced-CEC montmorillonite preparation approaches, ${ }^{21,17}$ as described before. ${ }^{26,27}$ The reduced CEC values of the modified MMTs were estimated by methylene-blue adsorption ${ }^{28}$ in the hydrated $\mathrm{Li}$ form, and more precise CEC values were measured by thermogravimetric analysis (TGA) after cationic exchange with alkyl-ammonium surfactants (vide infra); the reduced CEC values were found to be 1.37 and 1.05 mequiv/g (TGA). It should be noted that reducing the CEC of montmorillonites, as above, yields surfaces with varied surface charge (varied CEC) but with identical geometries and almost identical crystal structures $^{26}$ (there are very slight crystal lattice deformations due to permanent $\mathrm{Li}^{+}$migration within the alumino-silicate layered crystal, in the case of PGV, primarily by insertion within the $\mathrm{SiO}_{x}$ tetrahedra). This approach provides an excellent basis for comparing dynamics between systems with dielectric spectroscopy, vide infra, since the only parameter varied is the inorganic surface charge density.

The standard two-solvent cation exchange process was employed to intercalate alkylammonium surfactants between montmorillonite plates: ${ }^{29}$ First, octadecylammonium surfactants were obtained by protonating octadecylamine surfactants $\left(\mathrm{CH}_{3}\left(\mathrm{CH}_{2}\right)_{16} \mathrm{CH}_{2} \mathrm{NH}_{2}\right.$, purchased from Sigma Aldrich) with a stoichiometric quantity of hydrochloric acid $(\mathrm{HCl})$ in ethanol solution. The solution was stirred for $30 \mathrm{~min}$, and the temperature was increased to $70{ }^{\circ} \mathrm{C}$. In a second beaker, MMT clay particles (PGV, Nanocor Inc.) were dispersed in deionized water $(20 \mathrm{M} \Omega)$ and the suspension was stirred for 30 min at $70{ }^{\circ} \mathrm{C}$, resulting in a stable suspension with no visible clay aggregates. The surfactant solution was added to the clay suspension in the first beaker, and flocculation of excess surfactant (approximately $150 \%$ of the CEC) and white organoclay precipitation were observed immediately upon mixing. The reaction was allowed to completion under vigorous mixing for $2 \mathrm{~h}$, before the modified clay was paper-filtered and washed three times by ethanol using a Büchner funnel, after which all excess surfactant (surfactant not Coulombically bound on the MMT) was removed, as indicated by a clear silvernitrate test. A commercial alkyl-ammonium montmorillonite (Nanomer I.30E, CEC 1.45 mequiv/g) was used as received without further purification, as a control. Powders were dried in a vacuum oven for $12 \mathrm{~h}$ at $80{ }^{\circ} \mathrm{C}$, and kept in a desiccator, with desiccant and $\mathrm{P}_{2} \mathrm{O}_{5}$. The dried intercalated powders consist of fine montmorillonite grains.

Instrumentation. Thermogravimetric analysis (TGA) was performed on a Thermal Analysis (TA) Instruments SDT Q600. The temperature ramps were from 20 to $1000{ }^{\circ} \mathrm{C}$ under a heating rate of $10{ }^{\circ} \mathrm{C} / \mathrm{min}$, in a nitrogen environment.

Broadband dielectric relaxation spectroscopy (DRS) measurements were carried out isothermally by using a Novocontrol Alpha analyzer in a broad frequency $\left(10^{-2}\right.$ to $\left.10^{7} \mathrm{~Hz}\right)$ and temperature $\left(-140\right.$ to $\left.220{ }^{\circ} \mathrm{C}\right)$ range. The MMT/surfactant powders were pelletized by applying a $5 \mathrm{t}$ load on a $12 \mathrm{~mm}$ diameter die (inside an IR-sample mold). The disk-like specimens, of about $0.5 \mathrm{~mm}$ thick, were sandwiched between gold coated brass electrodes and mounted on a ZGS Alpha active sample cell, which was connected to a Quatro Cryosystem for temperature stabilization.

Prior to the dielectric measurements, the samples were dried at $60{ }^{\circ} \mathrm{C}$ under a vacuum for $8 \mathrm{~h}$ (an approach exceeding the industrial standards for "drying" these silicates prior to nanocomposite formation) and, thereafter, were additionally equilibrated at $110^{\circ} \mathrm{C}$ inside the dielectric cell for half an hour, to eliminate contributions in the data arising from the evaporation of free water and polarization effects. Measurements were performed isothermally from 110 to $-140{ }^{\circ} \mathrm{C}$ and sequentially from -140 to $220{ }^{\circ} \mathrm{C}$. At each temperature, samples were equilibrated for $20 \mathrm{~min}$ (temperature fluctuations $<0.2{ }^{\circ} \mathrm{C}$ ) prior to the frequency scans. The dielectric spectra were identical for the same temperatures in the cooling and heating cycles, indicating the absence of water evaporation. Beyond the two reduced-CEC (reduced surface charge density) MMTs, the dielectric properties of a commercial organo-MMT sample (based on the same-lot original PGV montmorillonite, with nominal CEC 1.45 mequiv/g) and the dielectric response of the original surfactant-free MMT (non-organically exchanged PGV) nanoparticles were also measured under the same conditions, and are reported here and compared against the reduced-CEC montmorillonites. 


\section{RESULTS}

The weight loss curves, determined by thermogravimetric analysis, and the corresponding first derivative curves are summarized in Figure 1 for all samples. At temperatures lower

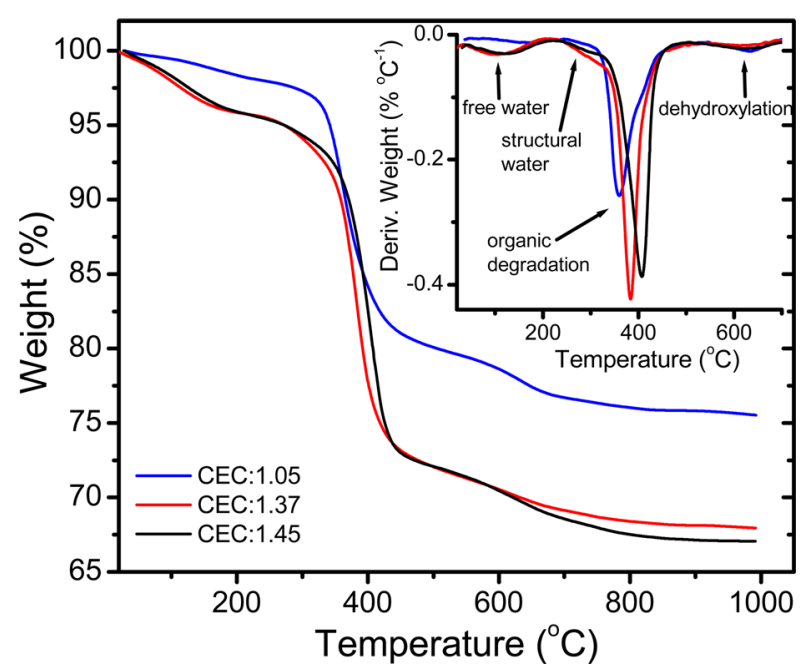

Figure 1. TGA curves (in nitrogen atmosphere) of the $C_{18}$ alkylammonium surfactants intercalated in montmorillonite of varied CEC values; the 1.45 mequiv/g CEC is a commercial alkyl-ammonium montmorillonite. (inset) The corresponding first derivative plot (differential weight loss, DTGA).

than the decomposition of the alkyl tails (abrupt drop around $400{ }^{\circ} \mathrm{C}$ ), the weight loss is related to the intergallery free water. In this temperature region, two processes manifest in all the samples, which are highlighted in the inset graph. The first process is revealed by the peaks in the temperature range from 40 to $230{ }^{\circ} \mathrm{C}$, whereas the second process corresponds to the shoulders that start at $200{ }^{\circ} \mathrm{C}$ and extend to temperatures overlapping the organic degradation at temperatures higher than $330{ }^{\circ} \mathrm{C}$. This dual step water loss is a common feature in the thermal behavior of hydrated smectites, ${ }^{30}$ as well as of organic/smectite complexes.

Double endothermic peaks have been observed in this temperature range, arising from the loss of free interlayer water (physisorbed intercalated water) and followed, at higher temperatures, by loss of structural water (water coordinated to the silicate surfaces and to the cations). ${ }^{21,30-32}$ For these layered nanoparticles, complete dehydration occurs well above $300{ }^{\circ} \mathrm{C}$, and practically a dry state at ambient conditions is not possible to be maintained due to the hydrophilic nature of the silicates. ${ }^{21}$ In our systems, the precise amount of water, which is allocated at free and strongly/weakly coordinated states, ${ }^{33}$ cannot be accurately quantified from the TGA plots due to the common temperature range of the surfactant degradation and the water evaporation. At higher temperatures, $400-600{ }^{\circ} \mathrm{C}$, MMT dehydroxylation involving the hydroxyl group removal from the silicate edges ${ }^{34}$ (and to a lesser degree from the $\mathrm{Al}$ octahedrals) takes place, and finally, the residual values at 1000 ${ }^{\circ} \mathrm{C}$ indicate the inorganic fraction, thus enabling the estimation of the CEC values by normalizing with the surfactant's molecular weight, after accounting for the free water and dehydroxylation weight losses. This clearly indicates that the CEC values reported in Figure 1 are overestimated by the amount of structural water, a common practice for CEC measurement through TGA, an overestimation that corre- sponds to a couple of percent of error in CEC. ${ }^{17}$ Typical "drying" (at $60{ }^{\circ} \mathrm{C}$ under a vacuum) done prior to dielectric measurements efficiently removes most of the free water (i.e., the sub-250 ${ }^{\circ} \mathrm{C}$ weight loss in the TGA above) but not the structural water.

Permittivity Formalism. A comparison plot of the dielectric dispersions at $60{ }^{\circ} \mathrm{C}$ for the alkylammonium surfactants confined in MMT plates of two different CEC values is presented in Figure 2. The imaginary part $\varepsilon^{\prime \prime}(f)$ of the
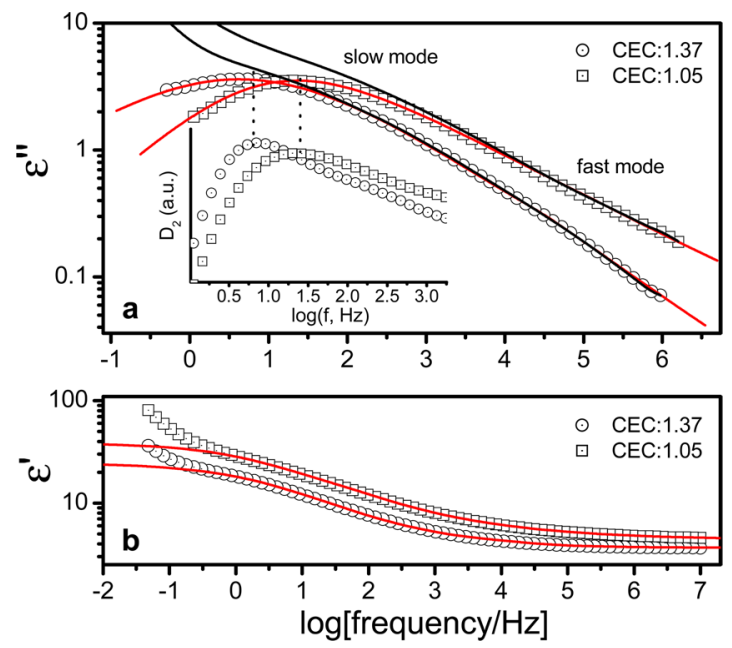

Figure 2. (a) Imaginary part of permittivity at $60{ }^{\circ} \mathrm{C}$ for the alkylammonium surfactants confined in MMT plates of two different CEC values. Both spectra consist of a broad relaxation with two components, a fast mode (FM) at high frequencies and a slow mode (SM) at lower frequencies. The symbols correspond to the experimental data after subtracting the low frequency dispersions from the raw data (black lines). The frequencies of the peak maximum, derived from the fitting curves (red lines), are in good agreement with the peak maximum in the logarithmic derivatives of the raw data (inset plot). The dotted lines are guides to the eyes. (b) Real part of permittivity and the corresponding fitting curves. The low frequency dispersion is pronounced in both samples by the upturn of the experimental data in the low frequency region.

complex dielectric function, $\varepsilon^{*}(f)=\varepsilon^{\prime}(f)-i \varepsilon^{\prime \prime}(f)$, for both samples is denoted by the black solid lines and consists of a broad bimodal relaxation process. The fast mode (FM) component is relaxing at high frequencies and appears as a shoulder of the slow mode (SM) component. At frequencies lower than the SM, a linear divergence is present and dominates the dielectric response. This contribution, which is also pronounced in the real part of permittivity, is related to the low frequency dispersion ${ }^{22,35-40}$ (LFD), a common feature in a variety of materials containing charge carriers such as solid ionic conductors, porous zeolites, layered mica, etc. This effect is attributed to space charge regions (islands) of ions being entrapped within heterogeneities or interfaces in the material, resulting in a discontinuity in their translational motion. The polarization of these regions gives rise to power law $\left(\sim f^{-s}\right)$ dielectric dispersions in the low frequency regime. Commonly, the exponent $s$ is taking values in the range $0.5<s<0.9$ and, thus, is indicating an enormous increase in both real and imaginary parts of the dielectric function, without reaching a direct current $\left(\mathrm{dc}\right.$ ) conductivity $^{36}$ (which is described by a steady state charge distribution). This dispersion arises from the inefficiency of the cations to diffuse in the bulk, is well 

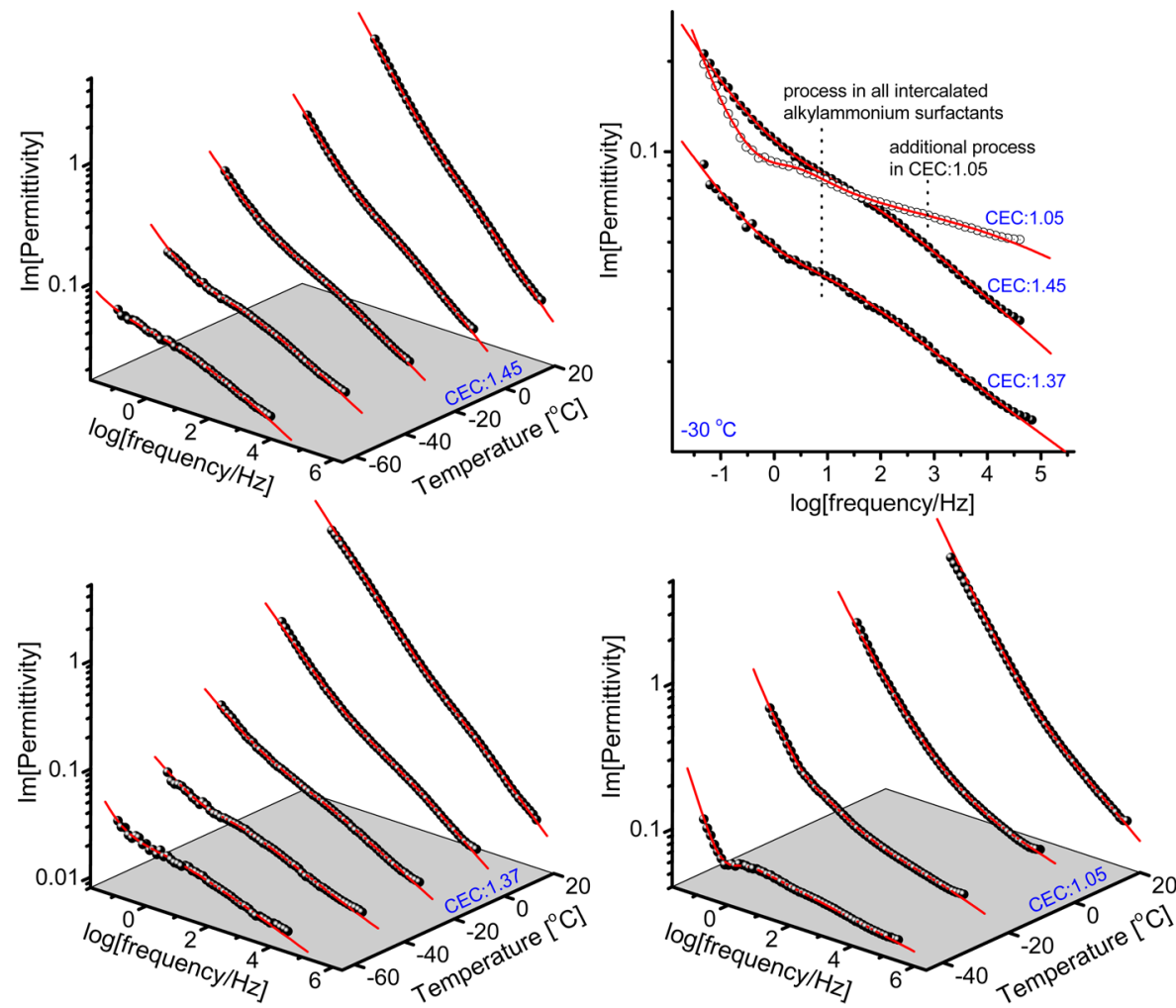

Figure 3. Summarized dielectric losses and the corresponding fitting curves of the fast mode component, for the alkylammonium intercalated samples, at low temperatures in steps of $20^{\circ} \mathrm{C}$. A comparison plot of the dielectric losses at $-30{ }^{\circ} \mathrm{C}$ is shown on the right top plot. The CEC1.45 and CEC1.37 samples exhibit one relaxation process with similar dynamics, whereas two relaxation processes are present in the CEC1.05 sample.

established in the literature, and is not further discussed in this work, which is focusing on the interlayer relaxation processes.

After subtracting the LFD, ${ }^{22,38,39,41-43}$ the optimum leastsquares fitting to the experimental data (red lines in Figure 2) was obtained by a superposition of two Havriliak-Negami (HN) expressions ${ }^{44-48}$

$$
\varepsilon^{*}(\omega)=\varepsilon_{\infty}+\sum_{i=1}^{2} \frac{\Delta \varepsilon_{i}}{\left[1+\left(i \omega \tau_{0, i}\right)^{1-\alpha_{i}}\right]^{\beta_{i}}}
$$

The fitting was performed by sharing the common parameters $\Delta \varepsilon_{i}, \tau_{0, i}, \alpha_{i}$, and $\beta_{i}$ in the real

$$
\begin{aligned}
& \varepsilon^{\prime}(\omega)=\varepsilon_{\infty} \\
& \quad+\sum_{i=1}^{2} \Delta \varepsilon_{i} \frac{\cos \left(\beta_{i} \phi_{i}\right)}{\left[1+2\left(\omega \tau_{0, i}\right)^{1-\alpha_{i}} \sin \left(\frac{1}{2} \alpha_{i} \pi\right)+\left(\omega \tau_{0, i}\right)^{2\left(1-\alpha_{i}\right.}\right)^{\beta_{i} / 2}}
\end{aligned}
$$

and, concurrently, in the imaginary part

$$
\varepsilon^{\prime \prime}(\omega)=\sum_{i=1}^{2} \Delta \varepsilon_{i} \frac{\sin \left(\beta_{i} \phi_{i}\right)}{\left[1+2\left(\omega \tau_{0, i}\right)^{1-\alpha_{i}} \sin \left(\frac{1}{2} \alpha_{i} \pi\right)+\left(\omega \tau_{0, i}\right)^{2\left(1-\alpha_{i}\right)}\right]^{\beta_{i} / 2}}
$$

of the complex permittivity function, with

$$
\varphi_{i}=\arctan \left[\frac{\left(\omega \tau_{0, i}\right)^{1-\alpha_{i}} \cos \left(\alpha_{i} \pi / 2\right)}{1+\left(\omega \tau_{0, i}\right)^{1-\alpha_{i}} \sin \left(\alpha_{i} \pi / 2\right)}\right]
$$

A power law expression $\left(\sigma_{0} / \varepsilon_{0}\right) \omega^{-s}$, describing conductivity effects, was added in the imaginary part (eq 3 ) when contribution from conductivity was present in the data sets.
In all above equations, $\omega(=2 \pi f)$ stands for the angular frequency; $\Delta \varepsilon=\varepsilon_{s}-\varepsilon_{\infty}$ is the relaxation strength, with $\varepsilon_{s}$ and $\varepsilon_{\infty}$ being the low and high frequency limits of the real part of permittivity; $\alpha$ and $\beta$ are the shape parameters associated with the symmetrical and asymmetrical broadening of each mode $(0<(1-\alpha) \beta, s \leq 1)$; and $\tau_{0}\left(=1 / 2 \pi f_{0}\right)$ is a characteristic time $\left(\omega \tau_{0}=1\right)$ which relates to each mode's maximum frequency $\left(f_{\max }\right)$ :

$$
f_{\max , i}=f_{0, i}\left[\frac{\sin \left[\left(1-a_{i}\right) \pi /\left(2+2 \beta_{i}\right)\right]}{\sin \left[\left(1-a_{i}\right) \beta_{i} \pi /\left(2+2 \beta_{i}\right)\right]}\right]^{1 /\left(1-\alpha_{i}\right)}
$$

For clarity, in the inset plot of Figure 2a, the logarithmic derivatives ${ }^{49}$ of the dielectric losses, manifesting the slopes in the $\varepsilon^{\prime \prime}(f)$ function $D_{2}=\partial \log \left[\varepsilon^{\prime \prime}(\omega)\right] / \partial \log (\omega)$, are also presented and found to exhibit maxima in good agreement with the fitting analysis. The SM slow mode depends on the surface charge density of the MMT and exhibits faster dynamics for the lower CEC silicates. This mode relaxes at higher frequencies than the conductivity contribution and can be ascribed to an induced polarization on the silicate surface, due to the mobility of the intercalated ammonium groups. This supposition is consistent with the high values in the dielectric losses and in the real part of permittivity, which is presented in Figure $2 \mathrm{~b}$. Moreover, the low frequency limit of the real part of permittivity, $\varepsilon_{s}$ increases when CEC decreases. This indicates the ability of the cations to better rearrange (more oriented dipole moments) on the silicate surfaces when spaced farther apart from each other (lower CEC), and it is also in good agreement with the faster dynamics observed for this sample (Figure 2a). Solid-state ${ }^{13} \mathrm{P}$ NMR spectroscopy on intercalated phosphonium based surfactants ${ }^{25}$ identified the longitudinal 
relaxation times of the cations to be around $1 \mathrm{~s}$ at room temperature, which is consistent with the time scale of the SM in this study.

Faster dynamics of polymeric chains in the same confined geometries have also been reported, ${ }^{23-25}$ although in this work they cannot be detected directly in the alkyls, due to the nonpolar nature of the alkyl chains here. It should be noted that in the samples with CEC 1.05 and 1.37 there is a direct, one-toone, correspondence between the surfactant population and the surface charge density (CEC) value. The observed shift in the dynamics above is qualitatively different from the trends reported in surfactant-excess samples, ${ }^{25}$ where, by increasing the organic population-overexchanging beyond the CECthe dynamics become faster; these faster dynamics are usually attributed to the lowering of the surface electrostatic potential (caused by the additional positive charge of the excess surfactant headgroups).

A decoupling in the time scale of the fast (FM) and the more intense slow (SM) modes is observed as the temperature is varied, due to the different activation energies associated with each process. Given the frequency window of the DRS instrument, the dynamics of the SM were followed and analyzed for higher temperatures $\left(30\right.$ to $\left.220^{\circ} \mathrm{C}\right)$, whereas the FM dynamics were followed in a lower temperature range $(-60$ to $30^{\circ} \mathrm{C}$ ). In Figure 3, the summarized dielectric losses for the FM, and the corresponding fitting curves, are presented for selected temperatures. For the fitting analysis of the CEC 1.37 and 1.45 samples, a superposition of a HN expression (for the dipolar contribution) and a conductivity power law term ${ }^{44-48}$ (for the linear increase in the low frequency region) were employed. Both samples exhibit similar time scales for the FM mode, although the CEC1.37 system is comprised exclusively of surface grafted surfactants and the CEC1.45 includes also a fraction of intercalated but unbound (confined but not surface tethered) surfactants. The CEC1.45 ("as received" organoMMT sample) is characterized by higher dielectric loss values and different dynamics, which may be attributed to electrostatic and interlayer polarization effects, originating from the presence of unbound surfactant. Interestingly, by decreasing the CEC value to 1.05 , two relaxation processes are clearly identified, although only the one located at higher frequencies can be followed and analyzed in the entire temperature range (due to the apparent merging of the two modes with increasing temperature). The analysis was carried out as described above, with the addition of one more HN expression, where needed. In the current work, we report the analysis results of the high frequency component, which could be followed in a broader temperature range.

Electric Modulus Formalism. In order to better obtain the relaxation map of the SM and FM in Figure 2 over a broad temperature range, the electric modulus formalism was also employed. The electric modulus is defined as the reciprocal complex permittivity ${ }^{50}$

$$
\begin{aligned}
M^{*}(\omega) & =\frac{1}{\varepsilon^{*}(\omega)} \\
& =M^{\prime}(\omega)+i M^{\prime \prime}(\omega) \\
& =\frac{\varepsilon^{\prime}(\omega)}{\varepsilon^{\prime 2}(\omega) \varepsilon^{\prime \prime 2}(\omega)}+i \frac{\varepsilon^{\prime \prime}(\omega)}{\varepsilon^{\prime 2}(\omega) \varepsilon^{\prime \prime 2}(\omega)}
\end{aligned}
$$

and is utilized to suppress conductivity and polarization phenomena and, in this manner, to better demonstrate the dipolar contributions. Macedo et al. ${ }^{51-53}$ applied this formalism to ionic conductors, modeling the dielectric response by an equivalent electric circuit of parallel capacitance and conductance elements. The conductivity relaxation time that corresponds to the diffusion of the ions in the material is expressed as

$$
\tau_{\sigma}=\varepsilon_{0} \varepsilon_{\mathrm{s}} / \sigma_{\mathrm{dc}}, \quad \mathbf{E}=\mathbf{E}_{\mathbf{0}} \varphi(t)=\mathbf{E}_{\mathbf{0}} \exp \left(-t / \tau_{\sigma}\right)
$$

and describes the decay of the electric field, E, when the electric displacement vector, $\mathbf{D}$, remains constant. In real conductors, the single relaxation time model can be extended to a distribution of relaxation times ${ }^{51}$ and the decay of the electric field is expressed by the Kohlrausch-Williams-Watts (KWW, stretched exponential) function ${ }^{50,51}$

$$
\varphi(t)=\exp \left[-\left(t / \tau_{\mathrm{KWW}}\right)^{\beta}\right], \quad 0<\beta \leq 1
$$

The complex electric modulus in the frequency domain is the Fourier transform of the time derivative of the electric field decay function

$$
M^{*}(\omega)=M_{\infty}\left[1-\int_{0}^{\infty}-\frac{\mathrm{d} \varphi(t)}{\mathrm{d} t} \exp (-i \omega t) \mathrm{d} t\right]
$$

Tsangaris et al. ${ }^{54,55}$ resolved the imaginary and real part of the modulus for different distributions of relaxation times in the frequency domain. A Havriliak-Negami distribution is expressed as

$$
\begin{aligned}
& M^{\prime}(\omega)= \\
& \quad M_{\infty} M_{\mathrm{s}} \frac{\left[M_{\mathrm{s}} A^{\beta}+\left(M_{\infty}-M_{\mathrm{s}}\right) \cos \beta \varphi\right] A^{\beta}}{M_{\mathrm{s}}^{2} A^{2 \beta}+2 A^{\beta}\left(M_{\infty}-M_{\mathrm{s}}\right) M_{\mathrm{s}} \cos \beta \varphi+\left(M_{\infty}-M_{\mathrm{s}}\right)^{2}}
\end{aligned}
$$

and

$$
\begin{aligned}
& M^{\prime \prime}(\omega)= \\
& \quad M_{\infty} M_{\mathrm{s}} \frac{\left[\left(M_{\infty}-M_{\mathrm{s}}\right) \sin \beta \varphi\right] A^{\beta}}{M_{\mathrm{s}}^{2} A^{2 \beta}+2 A^{\beta}\left(M_{\infty}-M_{\mathrm{s}}\right) M_{\mathrm{s}} \cos \beta \varphi+\left(M_{\infty}-M_{\mathrm{s}}\right)^{2}}
\end{aligned}
$$

where $M_{s}=1 / \varepsilon_{s}$ and $M_{\infty}=1 / \varepsilon_{\infty}$ and

$$
A=\left[1+2\left(\omega \tau_{0}\right)^{1-\alpha} \sin (\alpha \pi / 2)+\left(\omega \tau_{0}\right)^{2(1-\alpha)}\right]^{1 / 2}
$$

The argument $\varphi$ and the characteristic time $\tau_{0}$ are the same as those in eqs 4 and 5. A representative example of the frequency dependence of the modulus imaginary part at high temperatures is shown in Figure 4 for the CEC1.45 sample. Three processes can be clearly identified. In order of increasing frequency, i.e., from slower to faster process: The first process corresponds to the LFD in the permittivity formalism, and it is ascribed to space charge polarization effects. The second process dominates the spectra and is attributed to the SM, which is now shifted by about two decades of frequency higher than in the permittivity formalism. The third process is the FM, which is more pronounced in the modulus interpretation. This mode is well described by a Cole-Cole distribution $(\beta=1$ in eqs 10 and 11) and the maximum frequencies $f_{M_{\max }^{\prime \prime}}$ and $f_{\varepsilon_{\max }^{\prime \prime}}$ are closely positioned due to the weak relaxation strength of this mode. Since the modulus peak is located at ${ }^{54,56-58}$

$$
(\omega \tau)_{M_{\max }^{\prime \prime}}^{(1-\alpha)}=\varepsilon_{\mathrm{s}} / \varepsilon_{\infty}=M_{\infty} / M_{\mathrm{s}}
$$

and taking into account that the $M_{\infty}$ and $M_{s}$ values are comparable, the peaks in both formalisms are closely located at 


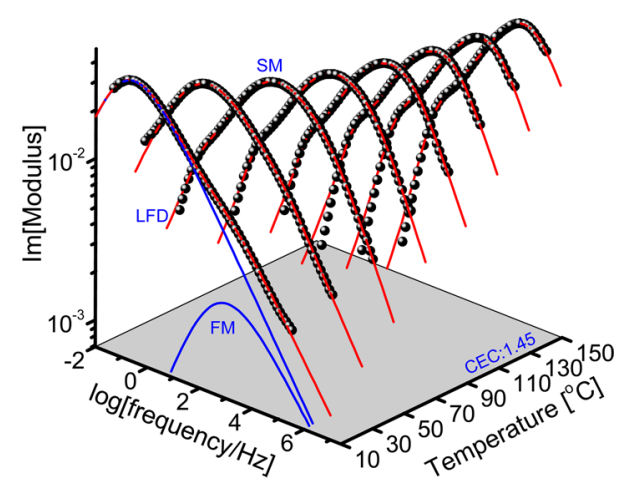

Figure 4. Frequency dependence of the imaginary part of the electric modulus for the CEC 1.45 sample at temperatures between 10 and 150 ${ }^{\circ} \mathrm{C}$ in steps of $20^{\circ} \mathrm{C}$. In order of increasing frequency, three modes are observed, originating from polarization effects due to the LFD, the slow mode (SM), and the fast mode (FM), with the last two modes merging at high temperatures.

$(\omega \tau)_{M_{\max }^{\prime \prime}}^{(1-\alpha)} \approx 1=(\omega \tau)_{\varepsilon_{\max }^{\prime \prime}}$. Notably, with increasing temperature, the dynamics of the SM can be distinct in two regimes, due to different shifting rates toward high frequencies. As a result, a change in the activation energy of this mechanism occurs, followed by merging with the FM relaxation in the high temperature limit. The same dependence is evident in the permittivity formalism, although less pronounced. Dielectric measurements were also performed on the corresponding surfactant-free $\left(\mathrm{Li}^{+}\right.$PGV montmorillonite without organic modification) samples to trace the origin of the FM. An indicative plot of the dielectric losses for selected temperatures is shown in Figure 5 for the CEC1.37 sample.

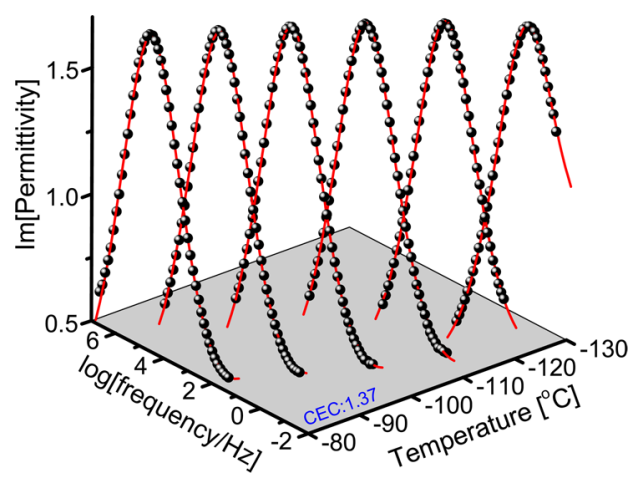

Figure 5. Dielectric losses, $\varepsilon^{\prime \prime}(f, T)$, of the surfactant-free CEC1.37 sample. The lines correspond to the best fitting of eq 1 to the experimental data. The temperature step is $10^{\circ} \mathrm{C}$.

The data analysis was preferably performed in the permittivity formalism, which has a more straightforward physical meaning. ${ }^{59-61}$ However, at high temperatures, the weak FM is masked by polarization effects on the silicate surface and analysis in the modulus formalism was employed to follow this mode in these cases.

\section{DISCUSSION}

The dynamics for the FM are summarized in Figure 6 as an Arrhenius plot of the modes' characteristic frequency (i.e., plot of the logarithm of the loss peak maximum frequency versus the reciprocal temperature). For low temperatures, below room temperature, the dynamics are analyzed in the permittivity formalism (open symbols), whereas, for high temperatures in the modulus formalism, because the FM becomes more evident and the fitting analysis more precise (filled symbols). As mentioned above, the shape parameters of the FM show no significant shift of the maximum frequency in the two formalisms (errors are within the symbol size). To conclusively assign the origins of this FM mode, measurements were performed also on the same silicate in the absence of intercalated surfactants (hydrated $\mathrm{Li}^{+} \mathrm{MMT}$ ), and these dynamics are also summarized in Figure 6. Prior to the DRS measurements, the samples were dried $\left(60^{\circ} \mathrm{C}, 8 \mathrm{~h}\right.$, vacuum) and equilibrated $\left(110^{\circ} \mathrm{C}, 0.5 \mathrm{~h}\right.$, in DRS cell) under the same experimental conditions as the alkyl-ammonium modified samples; this approach ensures efficient removal of the free/ unbound water from the silicate galleries.

The temperature dependence of the FM's maximum frequency $\left(f_{\max }\right)$ for both, intercalated and surfactant-free, samples can be described well by the Arrhenius equation $^{48,50,62-64}$

$$
f_{\max }(T)=f_{\infty} \exp \left(-\frac{\Delta E_{\mathrm{A}}}{k_{\mathrm{B}} T}\right)
$$

or the Eyring equation which has a similar form ${ }^{65-68}$

$$
f_{\max }(T)=\frac{k_{\mathrm{B}} T}{2 \pi h} \exp \left(\frac{\Delta S^{*}}{k_{\mathrm{B}}}\right) \exp \left(-\frac{\Delta H^{*}}{k_{\mathrm{B}} T}\right)
$$

In eq $14, \Delta E_{\mathrm{A}}$ is the activation energy, $f_{\infty}$ is the relaxation rate in the high temperature limit, and $k_{\mathrm{B}}$ is the Boltzmann's constant, whereas, in eq $15, \Delta S^{*}$ and $\Delta H^{*}$ denote the activation entropy and activation enthalpy, respectively, and $h$ is Planck's constant.

The $\Delta E_{\mathrm{A}}$ values, derived from the Arrhenius fit (eq 14) to the data, as well as the activation enthalpies (eq $15 \mathrm{fit}$ ), are similar in both intercalated and surfactant-free samples, despite the huge difference in relaxation times (about $10^{5}$ times faster dynamics in the surfactant-free systems, when comparing at the same temperature). Specifically, in order of decreasing CEC, $\Delta E_{\mathrm{A}}$ are $50 \pm 1,48 \pm 1$, and $48 \pm 2 \mathrm{~kJ} / \mathrm{mol}$ for the intercalated samples and $50 \pm 2,51 \pm 2$, and $47 \pm 2 \mathrm{~kJ} / \mathrm{mol}$ for the surfactant-free samples. These values are in good agreement with prior works ${ }^{69-73}\left(\Delta E_{\mathrm{A}} \sim 50 \mathrm{~kJ} / \mathrm{mol}\right)$ that studied the relaxation of confined water molecules in porous silica glasses and vermiculite silicates; thus, it is very reasonable to ascribe the observed dynamics to the reorientation of the intergallery water, which is confined between, and physisorbed on, the silicate surfaces. Since a precise calculation of the water content cannot be obtained, it is not feasible to delineate the water molecule spatial distribution on the surface. However, the obtained values compare well to the strength of hydrogen bonded water (e.g., water in ice has $\sim 19 \mathrm{~kJ} / \mathrm{mol}$ per H-bond, which manifests as $\Delta E_{\mathrm{A}} \sim 55 \mathrm{~kJ} / \mathrm{mol}$ for bulk ice, i.e., for dynamics which involve the simultaneous breaking of three hydrogen bonds ${ }^{70,74-76}$ ); this suggests, for our systems, the formation of a robust, almost fully hydrogen bonded, water monolayer with its hydrogens being coordinated primarily to the silicate surface oxygen atoms, or to other waters. Furthermore, for this confined relaxation mode, observed in both surfactant-modified MMT and in surfactant-free hydrated MMT, one can make a number of deductions: First, (a) from the similar activation energy $\Delta E_{\mathrm{A}}$ values (for the surfactantmodified MMT and the surfactant-free hydrated $\mathrm{Li}^{+} \mathrm{MMT}$ ) 


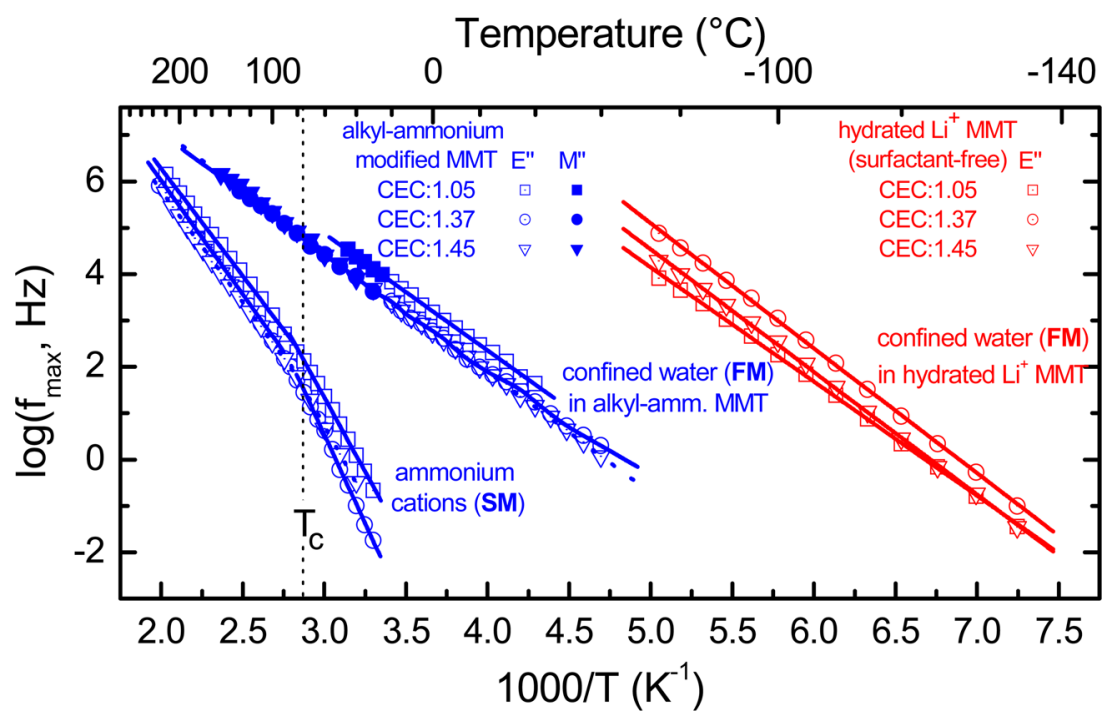

Figure 6. Cumulative Arrhenius plot of the relaxation frequencies (inverse of relaxation times) corresponding to the two modes of the systems: Intercalated end-tethered ammonium cations (SM) and confined water (FM). The relaxation of the ammonium cations (SM mode in Figure 2) exhibits a change in the dynamics around $T_{\mathrm{c}}=75^{\circ} \mathrm{C}$. Below $T_{\mathcal{O}}$, the surfactant SM dynamics are CEC-dependent, whereas, above $T_{\mathcal{O}}$ they are less sensitive to the CEC and asymptotically approach the water mode dynamics (FM mode in Figures 3 and 4). In the same plot, the Arrhenius dependence of the confined water relaxation frequency, measured in the respective varied-CEC hydrated montmorillonites (same silicates without any organic/surfactant modification, surfactant-free), is also presented. All three montmorillonites (varied CEC) are shown, over the entire temperature and frequency range; lines are best fits to the Arrhenius equation; open and filled symbols correspond to permittivity $\left(E^{\prime \prime}\right)$ and modulus $\left(M^{\prime \prime}\right)$ formalisms, respectively.

samples, it is strongly indicated that the relaxing molecules are the same, in this case, confined water (not only because it is the only common relaxing species in the two systems but also because the absolute values of $\Delta E_{\mathrm{A}}$ relate to the process of water hydrogen bond breaking and reforming under the same average coordination number). However, (b) the dynamics in the two systems, e.g., the modes' relaxation times, differ radically between the two systems, with the surfactant-free samples exhibiting much faster dynamics (about 10000 times faster dynamics than the corresponding surfactant-containing systems at the same temperature). This behavior denotes a high impediment of this relaxation mode by the presence of the surfactant molecules, and can be better explained in terms of the activation entropy $(\Delta S$, in eq 15). In the surfactant-free samples, an entropy gain can result from the increase of translational and configurational contributions for the nanoconfined water, which should be substantially larger than in the surfactant-modified systems, due to the absence of the hydrophobic alkyl groups as well as to a higher population of water located and relaxing farther away from the charged MMT surfaces (at loci of weaker electrostatic interactions and, thus, higher water mobilities). This entropy gain enters the relaxation time as an exponential factor, and small changes in $\Delta S$ can impose significant shifts in the relaxation times. (c) Additional evidence for the nature of these modes can be derived from the magnitude of relaxation times. The characteristic temperature at $100 \mathrm{~s}(1.6 \mathrm{mHz})$ associated with the glass transition temperature $\left(T_{\mathrm{g}}\right)$ and the pre-exponential factor $\left(f_{\infty}\right)$ correspond for the surfactant-free samples to $T_{\mathrm{g}} \sim 127 \mathrm{~K}$ and $f_{\infty} \sim 10^{-18}-10^{-20} \mathrm{~s}$, respectively, whereas, for the same surfaces when surfactant-modified, they correspond to $T_{\mathrm{g}} \sim 172 \mathrm{~K}$ and $f_{\infty} \sim 10^{-13}-10^{-14} \mathrm{~s}$. These values are very close to the characteristic dynamics of supercooled water in humid confined geometries ${ }^{77,78}$ and indicate a non-crystalline (supercooled) state of water for our systems. For example, in Figure 6, no change in the dynamics is shown at $273 \mathrm{~K}$, indicating no evident water crystallization in our systems, as is probably expected, since water crystallinity is prevented by the geometric constrainments.

In view of the above, we can formulate a better understanding for the correlation between the surfactant concentration, which is proportional to the CEC, and the dynamics of the confined water in Figure 3. The samples with similar CEC values of 1.45 and 1.37 mequiv/g have an identical distribution of relaxation times (despite the losses being significantly higher in the first sample). This indicates a similar structure in the hydrogen bond network, whereas the higher losses can be attributed to the higher conductivity of the CEC1.45 sample (most probably originating from its excess mobile surfactants). Interestingly, the sample with the lowest surfactant content (CEC1.05) exhibits a different distribution of relaxation times, compared to the other two CECs, showing a clearly bimodal relaxation (instead of a single broad mode, observed in the other two systems). This indicates the formation of a different hydrogen bond network: In this sample, the slower mode (at lower frequencies) exhibits similar dynamics as in the other two specimens (indicating that this originates from a water layer strongly bound on the silicate surfaces, as before). The faster mode (at higher frequencies) indicates a second type of water-weaker bound and more mobile than the previous, maybe a second water layer. However, the bimodal relaxation was not present in all temperatures, suggesting a possible rearrangement of the water molecules, or merging of the two modes. The dynamics of the faster mode were evident in the entire temperature range and are presented in Figure 6.

In Figure 6, along with the time scale of the confined water, the ammonium headgroup dynamics are also presented for all samples. Two distinct regimes can be observed for temperatures above and below $T_{\mathcal{O}}$ at $75{ }^{\circ} \mathrm{C}$ approximately. At temperatures higher than $T_{\mathcal{O}}$ the dynamics do not depend on 
the CEC value of the surfaces, and asymptotically merge with the dynamics of the confined water, in the high temperature limit. A split in the dynamics is observed at temperatures lower than $T_{c}$. At these temperatures, the dynamics of the alkylammonium cations differentiate for the three different systems; this decoupling in time scale depends on the CEC, with the lower surface charge systems (CEC1.05) demonstrating the fastest dynamics and CEC1.37 the slowest. The CEC1.45 is comparable and slightly faster than CEC1.37, despite having a very similar CEC value; this is in concert with the assumption of excess unbound surfactant ${ }^{25}$ in the CEC1.45 system.

The activation energy for the ammonium headgroup dynamics, calculated from eq 14 , is significantly lower for the CEC1.05 (125 kJ/mol) compared to the CEC1.37 (150 kJ/ $\mathrm{mol}$ ), indicating lowering of the electrostatic barriers related to the cation migration. To illustrate this better, the potential distribution in the intergallery space can be considered. In terms of a double diffuse layer model, ${ }^{79}$ and taking into account the confined geometry and the low hydration level, it is intuitive to assume that the cation mobility will be restricted in a Stern layer $(\sim 1-2$ cation diameters in size, defined by the Debye length). The cations in this layer are strongly bound and effectively cannot desorb from the surface; however, in the presence of water and electric field tangential to the interface, it is possible to exhibit high surface mobility, ${ }^{80-84}$ which can be comparable in magnitude to the cation mobilities in bulk solution. The potential in this layer $\psi(x)$, as a function of the distance $x$ normal to the surface, can be approximated by taking into account the Poisson-Boltzmann distribution, defining a potential $\psi_{0}$ at the surface $(x=0)$ and simplifying for low surface potential $\left(k_{\mathrm{B}} T \gg z e \psi_{0}\right)$ and for one type of ions: ${ }^{79}$

$$
\psi(x)=\psi_{0} \mathrm{e}^{-\kappa x}, \quad \kappa=\left(z e^{2} n / \varepsilon \varepsilon_{0} k_{\mathrm{B}} T\right)^{1 / 2}
$$

where $\kappa$ is the Debye-Hückel parameter (Debye length: $x_{\mathrm{D}}=$ $1 / \kappa)$ and $z, e$, and $n$ stand for the ion valence, the elementary electric charge, and the ion number density, respectively. Accordingly, the electrostatic barrier for cation migration on the surface relates to the potential difference $\Delta U=\psi_{0} \mathrm{e}^{-\kappa x}-\psi_{0}$. Since the absolute value of the total cation charge density is equal to the surface charge density $\sigma_{0}$, the surface potential can be written as $\psi_{0}=\sigma_{0} / \varepsilon \varepsilon_{0} \kappa$, with the $\sigma_{0}$ being directly proportional to the CEC value ( $\sigma_{0}$ is actually equal to the CEC divided by the specific surface area of the material; i.e., $\sigma_{0}$ can be calculated on the basis of well-known material properties). Given the system crystal chemistry and partial charges, the cations are primarily bound to the oxygen atom rings on the cleavage surface; thus, tangential migration may occur in the inner Helmholtz plane (at the same distance $x$ for both CECs). As a result, a capacitance can be ascribed to this layer with the electric field being approximately constant within. In this case, the exponential in the potential difference can be expanded in linear terms, and thus, $\Delta U$ scales with surface charge, or equivalently $\Delta U$ is directly proportional to the CEC. This is in excellent agreement with the observed activation energies, whose ratio is the same as the CEC ratio, within experimental uncertainty. In a more rigorous consideration, the strength of any hydrogen bonds should also be taken into account in the activation energy of the mobile charge carrier. Here, however, the hydrogen bonding can safely be ignored given the substantially higher electrostatic barriers and interactions.

The same characteristic frequency $f_{\max }$ is also associated with the cation hopping process atop the silicate surface, quantifying the average time required for the cation migration. In the low temperature region $\left(T<T_{\mathrm{c}}\right)$, the CEC1.05 sample demonstrated faster dynamics than the higher CEC systems, with the difference in dynamics between the two CECs becoming increasingly larger at lower temperature. This behavior is consistent with the lower surfactant density in CEC1.05, as well as with the weaker cation-surface charge interactions, ${ }^{85,86}$ compared to the other two systems; the same considerations also qualitatively predict the very similar dynamics observed in CEC1.45 and CEC1.37.

Furthermore, the ion hopping process would also manifest in the measured conductivity and intergallery polarization dynamics. According to the Barton-Nakajama-Namikawa $(\mathrm{BNN})$ relationship, ${ }^{87}$ the transition (onset) frequency, $f_{\mathcal{O}}$ for the time independent conduction, $\sigma_{\mathrm{dc}}$ (mean square displacement $\left.\left\langle r^{2}(t)\right\rangle \propto t\right)$, is proportional to the $\sigma_{\mathrm{dc}}$ value. Apparently, $f_{\mathrm{c}}$ and $f_{\max }$ are very closely positioned ${ }^{86,88,89}\left(\sigma(\omega)=\omega \varepsilon_{0} \varepsilon^{\prime \prime}(\omega)\right)$ and the faster dynamics of the CEC1.05 reveal also a more favorable tendency for long-range migration on the silicate surface. As expected, this is also confirmed by comparing the conductivity spectra (not shown here) with the CEC1.05 exhibiting higher $f_{\mathrm{c}}$ and higher conductivity values. It should be noted that, because of the confined geometry and the granular morphology of the investigated systems, a true dc conduction cannot occur; therefore, the term "tendency" is more appropriate here, and $\sigma_{\mathrm{dc}}$ is therefore defined as the conductivity value corresponding to the AC/LFD crossover frequency $f_{\mathrm{c}}$.

At temperatures higher than $T_{\mathcal{c}}$ the surfactant relaxations tend to merge with the dynamics of the confined water; the CEC dependence is less pronounced, and all systems are characterized by lower activation energies $(\sim 95 \mathrm{~kJ} / \mathrm{mol}$ calculated in the temperature range $80-140{ }^{\circ} \mathrm{C}$ ). A definitive explanation for this behavior is not obvious based on these DRS data alone. A possible mechanism that would account for this behavior would be a diffusion of hydrated cationic surfactants. In this case, the cations would be less coordinated to the silicate walls, would be solvated by (coordinated to) the water molecules, and would give rise to common dynamics for the water and the cations. This same mechanism would also permit for easier cation desorption from the walls (diffusion in the outer Helmholtz plane), and therefore would lower any surfacedictated potential barriers for the cation motion, cf. eq 16 . If the above supposition is correct, the presence of water determines the dynamics in this temperature range and also determines the crossover temperature $T_{\mathrm{c}} \sim 75{ }^{\circ} \mathrm{C}$, which indicates a transition from a hopping to a random-walk (Fickian) diffusive motion for the cations. This type of transition has been reported extensively from molecular modeling studies of cations, e.g., $\mathrm{Na}^{+}, \mathrm{Li}^{+}$, in hydrated smectites, ${ }^{90-92}$ as well as in PEO coordinated cations, ${ }^{20}$ albeit occurring at different temperatures, as expected. On the other hand, in surfactant-modified smectites and at temperatures very close to the value of $T_{c}$ observed here, alkyl group conformational transitions as well as changes in the thermal expansion of the gallery $d$-spacing have been reported. ${ }^{13,22}$ A correlation between these phenomena and the dynamics reported here is very possible, with the increase of the normal to the surface distance being attributed to the change in the alkyl group conformations at $T_{\mathrm{c}}$.

The experimental data, describing the dynamics of the ammonium cations in Figure 6, can also give a good fit to the Vogel-Tammann-Fulcher (VTF) equation. This approach would indicate cooperative dynamics, leading to an apparent 
glass transition of the alkane-based surfactants (in this case, the cations would follow the same dynamics). In good agreement with experimental ${ }^{14}$ and computer simulation ${ }^{10}$ results, differential scanning calorimetry (DSC) thermograms for these systems (not presented here) showed no evidence of a glasslike thermodynamic transition. According to Heinz et al., ${ }^{10}$ such phase transitions are related to the average packing density of the surfactants, which is defined as the ratio of the cross section over the total surface area per surfactant. For the CEC values of our samples (1.05-1.45 mequiv/g), the surfactant packing density is between 0.15 and 0.22 , and for these packings, a thermodynamic phase transition is not anticipated. Additional dielectric measurements were performed on the respective hydrated intercalated samples: Orders of magnitude faster dynamics were observed for the mobility of the cations, suggesting that the observed transition at $T_{c}$ is a kinetic transition that is strongly dependent on the physisorbed water. Moreover, due to the hydrophobic nature of the surfactant tails, if a glass-like thermodynamic transition was taking place, it would have been weakly dependent on the physisorbed water.

Assuming a Boltzmann factor for the probability of a vacant site formation, VTF dynamics can describe the mobility of the cations and, thus, interpret the characteristic temperature $T_{0}$ as the temperature where the cations are immobile. This would require strong interactions acting on the ions and, therefore, a "correlated" type of motion on the MMT surface. Such supposition is not supported by our data. In terms of the electric modulus formalism, the Kohlrausch exponent $\beta$ $\left(=1.14 / w\right.$, where $w$ is the $M^{\prime \prime}$ peak's full width at halfmaximum) of the electric field decay function ${ }^{93}$ is a measure of the ion interactions, and it is reported to increase with the cation separation distance. ${ }^{94}$ Here, fitting of eq 9 to the data indicates a different dependence with the $\beta$ values, with the CEC1.37 sample being significantly higher. This trend indicates that the motion of the ions is not "correlated" and that the exponent $\beta$ is mainly associated with the distribution of the vacant sites on the MMT surface (i.e., the CEC1.05 is characterized by broader $M^{\prime \prime}$ peaks or, equivalently, by smaller $\beta$ values, as would be expected due to the increase of the possible configurations of the ions).

Finally, a few comments can be made on the relevance of this work to the broader field of organically modified silicates and clays, as well as to the field of polymer/silicate nanocomposites. First, the existence of water, as well as the water effect on the surfactant dynamics, is qualitatively the same across the CECs studied and only differs quantitatively. This conclusion should be extrapolated with care to CEC values that are substantially different than the range probed here, i.e., 1.0-1.5 mequiv/g. For smaller CEC values (such as those characteristic of fluoromicas and hectorites), the surfactants are located much farther apart from each other, since CEC is directly proportional to surface charge density and, equivalently, to surfactant packing density. Thus, for the same surfactant size (molecular volume or alkyl chain length) and for sufficiently lower CECs, the surfactant molecules will be isolated from each other (no steric interactions or impingement with other surfactants on the same surface or on the opposite cleavage surface of the gallery). As a result, their dynamics are not expected to exhibit a transition $\left(T_{c}\right)$ but smoothly increase hopping frequency as the temperature increases; this has been seen before in simulations, and is probably what happens also here, for hydrated $\mathrm{Li}^{+}$montmorillonites. ${ }^{20}$ For higher CEC values (such as those characteristic of micas and vermiculites), the intermolecular steric effects will become more important (stronger geometric frustration, lower free volume), leading to an increase of the apparent $T_{\mathrm{g}}^{\mathrm{app}}$; also, in this case, an even stronger CEC dependence is expected for the dynamics below $T_{\mathrm{c}}$ (cf. Figure 6).

Beyond the CEC, also the chemistry of the surfactants is expected to affect the reported dynamics, probably in a nontrivial way. For example, this study was done exclusively on primary ammonium alkyls; it is expected that these behaviors will remain the same for some quaternary ammoniums, e.g., alkyl trimethyl ammoniums; however, it is very doubtful that other quaternary ammines, e.g., dialkyl dimethyl ammoniums or alkyl bis-hydroxyethyl ammoniums, will exhibit the same dynamics, given the differences in tethering configurations, headgroup geometry, and hydrophilicity. Furthermore, bigger changes in the surfactant chemistry, e.g., changing the ammonium headgroup for imidazolium ${ }^{95-97}$ or quinolinium, ${ }^{98}$ are expected to result in substantial qualitative changes in the surfactant dynamics from what is reported here. This expectation is not only because the electrostatic interactions and coordination on the surface will be altered (due to differences in head volume and geometry, partial charges, dipole moments, polarizability, etc.) but also because of the completely different hydration character of large polar cations compared to ammoniums. In fact, the use of alkyl-imidazolium surfactants for montmorillonite as a filler in PET nanocomposites is successful, primarily because imidazoliums are much more effective than ammoniums in displacing structural water from the montmorillonite intergallery. ${ }^{95,99}$ These are important considerations when extrapolating the current findings for industrial applications. Nevertheless, the results depicted in this study clearly demonstrate that the existence and dynamics of water in alkyl-ammonium modified silicates/ clays must be considered in the design of polymer nanocomposites, both to prevent degradation of hydroscopic polymers as well as to design the kinetics/processing of such composites.

\section{CONCLUSIONS}

Alkylammonium surfactants were Coulombically end-tethered and nanoscopically confined between montmorillonite plates of different surface charge density (i.e., of different cation exchange capacities, CECs). The reduction of CEC of montmorillonites yielded surfaces with varied surface charge but with identical geometries and crystal structures. The surfactants ionically exchanged the native positive cations, effectively becoming Coulombically end-tethered on the surfaces, and there was no excess or deficiency of charges. Although alkyl-cation exchange does render the montmorillonites miscible with polymers, they are typically assumed hydrophobic; measurable amounts of free and structural water were detected, even after standard drying. The dynamics of the alkyl-bearing cationic ammonium groups and of the physisorbed water on the silicate surfaces were probed by employing dielectric relaxation spectroscopy. The effects of CEC and of the confined water were found to be discrete, and governed the ammonium cation dynamics at different temperature ranges. A kinetic transition, manifested by a change in the activation energy of the cation hopping, was observed around $T_{\mathrm{c}} \sim 75^{\circ} \mathrm{C}$. For temperatures lower than $T_{\mathcal{O}}$ lower surface charge density resulted in faster dynamics and lower barriers for the mobility of the cations; this behavior is intuitively expected to be due to the increased number of possible configurations on the surface 
and due to the lower electrostatic potential on the silicate surface, respectively (entropic and enthalpic contributions). In terms of a Stern layer interpretation, the activation energies of the hopping mechanism were found to scale in good agreement with the CEC values, suggesting a tangential migration of the ammonium cations atop the surfaces, at the silicate/water interface, thus giving rise to a surface polarization. The activation barriers related to the migration process decreased for temperatures higher than $T_{\mathrm{c}}$ (cf. enthalpic contribution) and became similar between systems (viz., independent of the CEC surface charge). This change in the activation energy denotes a transition from a hopping-like (short-range) surfactant motion to a diffusive-type motion (at higher distances normal to the surface); the latter motion is inherently associated with the presence of water, exhibiting dynamics that are definitively correlated to the water dynamics. The last is evident by high temperature merging time scales for the water reorientation and the dynamics associated with the cation diffusion. The transition temperature $T_{\mathrm{c}}$ was found to be in good agreement with the temperature where conformational transitions of the alkyl groups have been reported for similar systems in the literature. Finally, in the low temperature range, the confined water was found to exhibit supercooled dynamics with an apparent glass transition temperature ( $T$ at a characteristic time of $100 \mathrm{~s}$ ), $T_{\mathrm{g}}^{\mathrm{app}}$ being 127 or $172 \mathrm{~K}$, depending on the confinement environments.

\section{AUTHOR INFORMATION}

\section{Corresponding Authors}

*E-mail: polyzosg@ornl.gov.

*E-mail:manias@psu.edu.

\section{Notes}

The authors declare no competing financial interest.

\section{ACKNOWLEDGMENTS}

This work was supported by the National Science Foundation (MWN grant, NSF Grant No. DMR-0602877); E.M.'s stay in AFRL was supported through an US Air Force SFFP fellowship, administered through NRC. Work was also supported by the U.S. Department of Energy, Office of Electricity Delivery and Energy Reliability - Power Electronics Program. Oak Ridge National Laboratory is operated for the U.S. Department of Energy by U.T.-Battelle under Contract No. DE-AC05-00OR22725.

\section{REFERENCES}

(1) Ray, S. S.; Okamoto, M. Polymer/layered Silicate Nanocomposites: A Review from Preparation to Processing. Prog. Polym. Sci. 2003, 28, 1539-1641.

(2) Vaia, R. A.; Giannelis, E. P. Polymer Melt Intercalation in Organically-Modified Layered Silicates: Model Predictions and Experiment. Macromolecules 1997, 30, 8000-8009.

(3) Manias, E.; Polizos, G.; Nakajima, H.; Heidecker, M. J. Flammability of Polymer Nanocomposites; Wiley \& Sons: NJ, 2007.

(4) Vaia, R. A.; Jandt, K. D.; Kramer, E. J.; Giannelis, E. P. Kinetics of Polymer Melt Intercalation. Macromolecules 1995, 28, 8080-8085.

(5) Manias, E.; Chen, H.; Krishnamoorti, R.; Genzer, J.; Kramer, E. J.; Giannelis, E. P. Intercalation Kinetics of Long Polymers in $2 \mathrm{~nm}$ Confinements. Macromolecules 2000, 33, 7955-7966.

(6) Heinz, H.; Castelijns, H. J.; Suter, U. W. Structure and Phase Transitions of Alkyl Chains on Mica. J. Am. Chem. Soc. 2003, 125, 9500-9510.
(7) Heinz, H.; Paul, W.; Suter, U. W.; Binder, K. Analysis of the Phase Transitions in Alkyl-Mica by Density and Pressure Profiles. J. Chem. Phys. 2004, 120, 3847-3854.

(8) Hackett, E.; Manias, E.; Giannelis, E. P. Molecular Dynamics Simulations of Organically Modified Layered Silicates. J. Chem. Phys. 1998, 108, 7410-7415.

(9) Heinz, H.; Vaia, R. A.; Krishnamoorti, R.; Farmer, B. L. SelfAssembly of Alkylammonium Chains on Montmorillonite: Effect of Chain Length, Head Group Structure, and Cation Exchange Capacity. Chem. Mater. 2007, 19, 59-68.

(10) Heinz, H.; Vaia, R. A.; Farmer, B. L. Relation Between Packing Density and Thermal Transitions of Alkyl Chains on Layered Silicate and Metal Surfaces. Langmuir 2008, 24, 3727-3733.

(11) Heinz, H. Clay Minerals for Nanocomposites and Biotechnology: Surface Modification, Dynamics and Responses to Stimuli. Clay Miner. 2012, 47, 205-230.

(12) Vaia, R. A.; Teukolsky, R. K.; Giannelis, E. P. Interlayer Structure and Molecular Environment of Alkylammonium Layered Silicates. Chem. Mater. 1994, 6, 1017-1022.

(13) Osman, M. A.; Seyfang, G.; Suter, U. W. Two-Dimensional Melting of Alkane Monolayers Ionically Bonded to Mica. J. Phys. Chem. B 2000, 104, 4433-4439.

(14) Osman, M. A.; Ploetze, M.; Skrabal, P. Structure and Properties of Alkylammonium Monolayers Self-Assembled on Montmorillonite Platelets. J. Phys. Chem. B 2004, 108, 2580-2588.

(15) Lagaly, G.; Dekany, I. Adsorption on Hydrophobized Surfaces: Clusters and Self-Organization. Adv. Colloid Interface Sci. 2005, 114, 189-204.

(16) Mohanambe, L.; Vasudevan, S. Aromatic Molecules in Restricted Geometries: Pyrene Excimer Formation in an Anchored Bilayer. J. Phys. Chem. B 2006, 110, 14345-14354.

(17) Theng, B. K. G. The Chemistry of Clay-Organic Reactions; Wiley: New York, 1975.

(18) Henn, F.; Devautour-Vinot, S.; Giuntini, J. C.; Maurin, G. Alkali Metal Bonding Energy and Activation Energy for DC Conductivity in Porous and Glassy Solid Oxides. J. Phys. Chem. B 2004, 108, 1393613943.

(19) Cajkovski, T.; Davidovic, M.; Pissis, P.; Polizos, G.; Cajkovski, D.; Likar-Smiljanic, V.; Biljic, R.; Nedic, Z.; Mioc, U. B. Dielectric Investigation of Electrical Conductivity in the Copper Salt of the 12Tungstophosphoric Acid Hydrate. Solid State Ionics 2003, 162, $203-$ 208.

(20) Kuppa, V.; Manias, E. Computer Simulation of PEO/LayeredSilicate Nanocomposites: 2. Lithium Dynamics in $\mathrm{PEO} / \mathrm{Li}^{+}$Montmorillonite Intercalates. Chem. Mater. 2002, 14, 2171-2175.

(21) Greene-Kelly, R. The Differential Thermal Investigation of Clays; Mineralogical Society: London, 1957.

(22) Jacobs, J. D.; Koerner, H.; Heinz, H.; Farmer, B. L.; Mirau, P.; Garrett, P. H.; Vaia, R. A. Dynamics of Alkyl Ammonium Intercalants Within Organically Modified Montmorillonite: Dielectric Relaxation and Ionic Conductivity. J. Phys. Chem. B 2006, 110, 20143-20157.

(23) Jeschke, G.; Panek, G.; Schleidt, S.; Jonas, U. Addressing the Interface in Polymer-Clay Nanocomposites by Electron Paramagnetic Resonance Spectroscopy on Surfactant Probes. Polym. Eng. Sci. 2004, $44,1112-1121$.

(24) Schleidt, S.; Spiess, H. W.; Jeschke, G. A Site-Directed SpinLabeling Study of Surfactants in Polymer-Clay Nanocomposites. Colloid Polym. Sci. 2006, 284, 1211-1219.

(25) Panek, G.; Schleidt, S.; Mao, Q.; Wolkenhauer, M.; Spiess, H. W.; Jeschke, G. Heterogeneity of the Surfactant Layer in Organically Modified Silicates and Polymer/Layered Silicate Composites. Macromolecules 2006, 39, 2191-2200.

(26) Komadel, P.; Madajova, J.; Bujdak, J. Preparation and Properties of Reduced-Charge Smectites - A Review. Clays Clay Miner. 2005, 53, 313-334.

(27) Komadel, P.; Hrobarikova, J.; Smrcok, L.; KoppelhuberBitschnau, B. Hydration of Reduced-Charge Montmorillonite. Clay Miner. 2002, 37, 543-550. 
(28) Bujdak, J.; Janek, M.; Madajova, J.; Komadel, P. Influence of the Layer Charge Density of Smectites on the Interaction with Methylene Blue. J. Chem. Soc., Faraday Trans. 1998, 94, 3487-3492.

(29) Polizos, G.; Tomer, V.; Manias, E.; Randall, C. A. Epoxy-Based Nanocomposites for Electrical Energy Storage. II: Nanocomposites with Nanofillers of Reactive Montmorillonite Covalently-Bonded with Barium Titanate. J. Appl. Phys. 2010, 108, 074117.

(30) Mackenzie, R. C. Differential Thermal Investigation of Clays; Mineralogical Society: London, 1957.

(31) Xie, W.; Gao, Z.; Pan, W. P.; Hunter, D.; Singh, A.; Vaia, R. Thermal Degradation Chemistry of Alkyl Quaternary Ammonium Montmorillonite. Chem. Mater. 2001, 13, 2979-2990.

(32) Bray, H. J.; Redfern, S. A. T.; Clark, S. M. The Kinetics of Dehydration in Ca-Montmorillonite: An in Situ X-Ray Diffraction Study. Mineral. Mag. 1998, 62, 647-656.

(33) Sposito, G.; Prost, R. Structure of Water Adsorbed on Smectites. Chem. Rev. 1982, 82, 553-573.

(34) Vangroos, A. F. K.; Guggenheim, S. Dehydroxylation of CaExchanged and $\mathrm{Mg}$-Exchanged Montmorillonite. Am. Mineral. 1989, $74,627-636$.

(35) Jonscher, A. K. Dielectric Relaxation in Solids; Chelsea Dielectric Press: London, 1983.

(36) Jonscher, A. K. The Universal Dielectric Response and its Physical Significance. IEEE Trans. Electr. Insul. 1992, 27, 407-423.

(37) Jonscher, A. K. Dielectric Relaxation in Solids. J. Phys. D: Appl. Phys. 1999, 32, R57-R70.

(38) Jeevanandam, P.; Vasudevan, S. Anomalous Low Frequency Dispersion and Dielectric Relaxation in the Layered Intercalated Compounds Cd(0.75) PS(3)A(0.5)(H2O) $[\mathrm{A}=\mathrm{K}, \mathrm{Cs}]$. J. Chem. Phys. 1998, 108, 1206-1215.

(39) Jeevanandam, P.; Vasudevan, S. Conductivity and Dielectric Response in the Ion-Exchange Intercalated Mono- and Double-Layer Hydrates Cd0.75PS3Na0.5(H2O)(y), y = 1, 2. J. Phys. Chem. B 1998, 102, 3082-3089.

(40) Kyritsis, A.; Siakantari, M.; Vassilikou-Dova, A.; Pissis, P.; Varotsos, P. Dielectric and Electrical Properties of Polycrystalline Rocks at Various Hydration Levels. IEEE Trans. Dielectr. Electr. Insul. 2000, 7, 493-497.

(41) Dissado, L. A.; Hill, R. M. Anomalous Low-Frequency Dispersion - Near Direct-Current Conductivity in Disordered LowDimensional Materials. J. Chem. Soc., Faraday Trans. 1984, 80, 291319.

(42) Jonscher, A. K. Low-Frequency Dispersion in CarrierDominated Dielectrics. Philos. Mag. B 1978, 38, 587-601.

(43) Tuncer, E. Signs of Low Frequency Dispersions in Disordered Binary Dielectric Mixtures (Fifty-Fifty). J. Phys. D: Appl. Phys. 2004, 37, 334-342.

(44) Boettcher, C. J. F.; Bordewijk, P. Theory of Electric Polarization; Elsevier: Amsterdam, The Netherlands, 1978.

(45) Havriliak, S.; Negami, S. A Complex Plane Analysis of AlphaDispersions in Some Polymer Systems. J. Polym. Sci., Polym. Symp. 1966, 14, 89-99.

(46) Alvarez, F.; Alegria, A.; Colmenero, J. Relationship Between the Time-Domain Kohlrausch-Williams-Watts and Frequency-Domain Havriliak-Negami Relaxation Functions. Phys. Rev. B 1991, 44, 7306-7312.

(47) Boersma, A.; van Turnhout, J.; Wübbenhorst, M. Dielectric Characterization of a Thermotropic Liquid Crystalline Copolyesteramide: 2. Orientation and Crystallinity. Macromolecules 1998, 31, 7461-7466.

(48) Kremer, F.; Schönhals, A. Broadband Dielectric Spectroscopy; Springer-Verlag: Berlin, 2002.

(49) Capaccioli, S.; Lucchesi, M.; Casalini, R.; Rolla, P. A.; Bona, N. Influence of the Wettability on the Electrical Response of Microporous Systems. J. Phys. D: Appl. Phys. 2000, 33, 1036-1047.

(50) McCrum, N. G.; Read, B. E.; Williams, G. Anelastic and Dielectric Effects in Polymeric Solids; Wiley: London, 1967.
(51) Macedo, P. B.; Moynihan, C. T.; Bose, R. Role of Ionic Diffusion in Polarization in Vitreous Ionic Conductors. Phys. Chem. Glasses 1972, 13, 171-179.

(52) Moynihan, C. T.; Boesch, L. P.; Laberge, N. L. Decay Function for Electric-Field Relaxation In-Vitreous Ionic Conductors. Phys. Chem. Glasses 1973, 14, 122-125.

(53) Ambrus, J. H.; Moynihan, C. T.; Macedo, P. B. Conductivity Relaxation in Concentrated Aqueous-Electrolyte Solution. J. Phys. Chem. 1972, 76, 3287-3295.

(54) Tsangaris, G. M.; Psarras, G. C.; Kouloumbi, N. Electric Modulus and Interfacial Polarization in Composite Polymeric Systems. J. Mater. Sci. 1998, 33, 2027-2037.

(55) Psarras, G. C.; Manolakaki, E.; Tsangaris, G. M. Electrical Relaxations in Polymeric Particulate Composites of Epoxy Resin and Metal Particles. Composites, Part A 2002, 33, 375-384.

(56) Wübbenhorst, M.; van Turnhout, J. Analysis of Complex Dielectric Spectra. I. One-Dimensional Derivative Techniques and Three-Dimensional Modeling. J. Non-Cryst. Solids 2002, 305, 40-49.

(57) Howell, F. S.; Moynihan, C. T.; Macedo, P. B. Electrical Relaxations in Mixtures of Lithium-Chloride and Glycerol. Bull. Chem. Soc. Jpn. 1984, 57, 652-661.

(58) Pathmanathan, K.; Johari, G. P. Dipolar and Conductivity Relaxations in LiCl-Propylene Glycol Systems. J. Chem. Phys. 1991, 95, 5990-5998.

(59) Elliott, S. R. Use of the Modulus Formalism in the Analysis of AC Conductivity Data for Ionic Glasses. J. Non-Cryst. Solids 1994, 170, 97-100.

(60) Sidebottom, D. L.; Green, P. F.; Brow, R. K. Scaling Parallels in the Non-Debye Dielectric Relaxation of Ionic Glasses and Dipolar Supercooled Liquids. Phys. Rev. B 1997, 56, 170-177.

(61) Roling, B.; Happe, A.; Funke, K.; Ingram, M. D. Carrier Concentrations and Relaxation Spectroscopy: New Information from Scaling Properties of Conductivity Spectra in Ionically Conducting Glasses. Phys. Rev. Lett. 1997, 78, 2160-2163.

(62) Havriliak, Jr. S.; Havriliak, S. J. Dielectric and Mechanical Relaxation in Materials; Hanser Verlag: Munich, Germany, 1997.

(63) Polizos, G.; Georgoussis, G.; Kyritsis, A.; Shilov, V. V.; Shevchenko, V. V.; Gomza, Y. P.; Nesin, S. D.; Klimenko, N. S.; Wartewig, S.; Pissis, P. Structure and Electrical Conductivity in Novel Polyurethane Ionomers. Polym. Int. 2000, 49, 987-992.

(64) Charnetskaya, A. G.; Polizos, G.; Shtompel, V. I.; Privalko, E. G.; Kercha, Y. Y.; Pissis, P. Phase Morphology and Molecular Dynamics of a Polyurethane Ionomer Reinforced with a Liquid Crystalline Filler. Eur. Polym. J. 2003, 39, 2167-2174.

(65) Glasstone, S. N.; Laidler, K.; Eyring, H. The Theory of Rate Process; McGraw-Hill: New York, 1941.

(66) Starkweather, H. W. Simple and Complex Relaxations. Macromolecules 1981, 14, 1277-1281.

(67) Kudlik, A.; Tschirwitz, C.; Blochowicz, T.; Benkhof, S.; Rössler, E. Slow Secondary Relaxation in Simple Glass Formers. J. Non-Cryst. Solids 1998, 235, 406-411.

(68) Capaccioli, S.; Casalini, R.; Lucchesi, M.; Lovicu, G.; Prevosto, D.; Pisignano, D.; Romano, G.; Rolla, P. A. Influence of the End Groups on Dynamics of Propylene Glycol Oligomers Studied by Wideband Dielectric Spectroscopy. J. Non-Cryst. Solids 2002, 307, $238-245$.

(69) Pissis, P.; Laudat, J.; Daoukaki, D.; Kyritsis, A. Dynamic Properties of Water in Porous Vycor Glass Studied by Dielectric Techniques. J. Non-Cryst. Solids 1994, 171, 201-207.

(70) Ryabov, Y.; Gutina, A.; Arkhipov, V.; Feldman, Y. Dielectric Relaxation of Water Absorbed in Porous Glass. J. Phys. Chem. B 2001, $105,1845-1850$.

(71) Frunza, L.; Kosslick, H.; Pitsch, I.; Frunza, S.; Schönhals, A. Rotational Fluctuations of Water Inside the Nanopores of SBA-Type Molecular Sieves. J. Phys. Chem. B 2005, 109, 9154-9159.

(72) Cerveny, S.; Schwartz, G. A.; Bergman, R.; Swenson, J. Glass Transition and Relaxation Processes in Supercooled Water. Phys. Rev. Lett. 2004, 93, 245702. 
(73) Spanoudaki, A.; Albela, B.; Bonneviot, L.; Peyrard, M. The dynamics of Water in Nanoporous Silica Studied by Dielectric Spectroscopy. Eur. Phys. J. E 2005, 17, 21-27.

(74) Auty, R. P.; Cole, R. H. Dielectric Properties of Ice and Solid D2O. J. Chem. Phys. 1952, 20, 1309-1314.

(75) Hasted, J. B. Aqueous Dielectrics; Chapman and Hall: London, 1973

(76) Chan, R. K.; Davidson, D. W.; Whalley, E. Effect of Pressure on the Dielectric Properties of Ice I. J. Chem. Phys. 1965, 43, 2376-2383.

(77) Bergman, R.; Swenson, J. Dynamics of Supercooled Water in Confined Geometry. Nature 2000, 403, 283-286.

(78) Swenson, J. The Glass Transition and Fragility of Supercooled Confined Water. J. Phys.: Condens. Matter 2004, 16, S5317-S5327.

(79) Delgado, A. V.; Arroyo, F. J. Interfacial Electrokinetics and Electrophoresis; Marcel Dekker: New York, 2001.

(80) Dukhin, S. S.; Zimmermann, R; Werner, C. A Concept for the Generalization of the Standard Electrokinetic Model. Colloids Surf., A 2001, 195, 103-112.

(81) Dukhin, A. S.; Vandeven, T. G. M. Electrokinetic Characterization of Polydisperse Colloidal Particles. J. Colloid Interface Sci. 1994, $165,9-18$.

(82) Lyklema, J.; Minor, M. On Surface Conduction and its Role in Electrokinetics. Colloids Surf., A 1998, 140, 33-41.

(83) Mangelsdorf, C. S.; White, L. R. Effects of Stern-Layer Conductance on Electrokinetic Transport-Properties of Colloidal Particles. J. Chem. Soc., Faraday Trans. 1990, 86, 2859-2870.

(84) Vlassiouk, I.; Rios, F.; Vail, S. A.; Gust, D.; Smirnov, S. Electrical Conductance of Hydrophobic Membranes or What Happens Below the Surface. Langmuir 2007, 23, 7784-7792.

(85) Wei, T. C.; Hillhouse, H. W. Ion Transport in the Microporous Titanosilicate ETS-10. J. Phys. Chem. B 2006, 110, 13728-13733.

(86) Garcia-Belmonte, G.; Bisquert, J. Entropy Factor in the Hopping Frequency for Ionic Conduction in Oxide Glasses Induced by Energetic Clustering. J. Chem. Phys. 2005, 123, 074504.

(87) Dyre, J. C.; Schröder, T. B. Universality of AC Conduction in Disordered Solids. Rev. Mod. Phys. 2000, 72, 873-892.

(88) Garcia-Belmonte, G.; Henn, F.; Bisquert, J. Dielectric Relaxation Strength in Ion Conducting Glasses Caused by Cluster Polarization. Chem. Phys. 2006, 330, 113-117.

(89) Polizos, G.; Shilov, V. V.; Pissis, P. Temperature and Pressure Effects on Molecular Mobility and Ionic Conductivity in Telechelics Based on Poly(Ethylene Oxide) Capped with Hydroxyl Groups at Both Ends. J. Non-Cryst. Solids 2002, 305, 212-217.

(90) Chang, F. R. C.; Skipper, N. T.; Sposito, G. ComputerSimulation of Interlayer Molecular-Structure in Sodium Montmorillonite Hydrates. Langmuir 1995, 11, 2734-2741.

(91) Chang, F. R. C.; Skipper, N. T.; Sposito, G. Monte Carlo and Molecular Dynamics Simulations of Interfacial Structure in LithiumMontmorillonite Hydrates. Langmuir 1997, 13, 2074-2082.

(92) Chang, F. R. C.; Skipper, N. T.; Sposito, G. Monte Carlo and Molecular Dynamics Simulations of Electrical Double-Layer Structure in Potassium-Montmorillonite Hydrates. Langmuir 1998, 14, 12011207.

(93) Ngai, K. L.; Martin, S. W. Correlation Between the Activation Enthalpy and Kohlrausch Exponent for Ionic-Conductivity in Oxide Glasses. Phys. Rev. B 1989, 40, 10550-10556.

(94) Patel, H. K.; Martin, S. W. Fast Ionic-Conduction in Na2S +B2S3 Glasses - Compositional Contributions to Nonexponentiality in Conductivity Relaxation in the Extreme Low-Alkali-Metal Limit. Phys. Rev. B 1992, 45, 10292-10300.

(95) Gilman, J. W.; Awad, W. H.; Davis, R. D.; Shields, J.; Harris, R. H.; Davis, C.; Morgan, A. B.; Sutto, T. E.; Callahan, J.; Trulove, P. C.; et al. Polymer/Layered Silicate Nanocomposites from Thermally Stable Trialkylimidazolium-Treated Montmorillonite. Chem. Mater. 2002, 14, 3776-3785.

(96) Wang, Z. M.; Chung, T. C.; Gilman, J. W.; Manias, E. Melt Processable Syndiotactic-Polystyrene/Montmorillonite Nanocomposites. J. Polym. Sci., Part B: Polym. Phys. 2003, 41, 3173-3187.
(97) Costache, M. C.; Heidecker, M. J.; Manias, E.; Gupta, R. K.; Wilkie, C. A. Benzimidazolium Surfactants for Modification of Clays for Use with Styrenic Polymers. Polym. Degrad. Stab. 2007, 92, 17531762.

(98) Costache, M. C.; Heidecker, M. J.; Manias, E.; Wilkie, C. A. Preparation and Characterization of PET/Clay Nanocomposites by Melt Blending Using Thermally Stable Surfactants. Polym. Adv. Technol. 2006, 17, 764-771.

(99) Davis, C. H.; Mathias, L. J.; Gilman, J. W.; Schiraldi, D. A.; Shields, J. R.; Trulove, P.; Sutto, T. E.; Delong, H. C. Effects of MeltProcessing Conditions on the Quality of Poly(Ethylene Terephthalate) Montmorillonite Clay Nanocomposites. J. Polym. Sci., Part B: Polym. Phys. 2002, 40, 2661-2666. 